\title{
Neural learning invariant to network size changes
}

\author{
Vicente Ruiz de Angulo and Carme Torras \\ Institut de Robòtica i Informàtica Industrial (CSIC-UPC), \\ Parc Tecnològic de Barcelona-Edifici U, Llorens i Artigas 4-6, 08028-Barcelona, Spain \\ ruiz@iri.upc.es, torraseiri.upc.es
}

\begin{abstract}
This paper investigates the functional invariance of neural network learning methods. By functional invariance we mean the property of producing functionally equivalent minima as the size of the network grows, when the smoothing parameters are fixed. We study three different principles on which functional invariance can be based, and try to delimit the conditions under which each of them acts. We find out that, surprisingly, some of the most popular neural learning methods, such as weight-decay and input noise addition, exhibit this interesting property.
\end{abstract}

\section{Introduction}

This work stems from an observation we made in analyzing the behaviour of a deterministic algorithm to emulate neural learning with random weights. We found that, for a fixed variance greater than zero, there is a number of hidden units above which the learned function does not change, or the change is slight and tends to zero as the size the network grows [7]. Here we study the conditions a neural learning algorithm should satisfy in order to lead to the same function, irrespective of network size.

Methods for complexity reduction [1] usually include one parameter (and sometimes more than one) to regulate the simplicity or smoothness imposed on the function implemented by the network. Each method simplifies the network in a way that is supposed to be optimal for the class of functions that is being approximated. Thus, ideally, the optimal level of smoothing should be obtained only by manipulating the above-mentioned parameter. Variability caused by other sources must be considered spurious uncertainty. For example, functionally different minima obtained by the same algorithm when running on different architectures or when departing from different initial points are embarrassing for the practitioner, who would desire to be freed from having to optimize the algorithm also along these lines. In particular, the selection of the number of hidden units of the architecture can influence decisively the result and is computationally cumbersome.

This motivates the interest in complexity minimization methods that show dependence only on the explicit complexity parameter and not on the size of the chosen architecture. However, there are no claims about functional invariance for the known methods, although Neal [4] devised a prior such that the complete bayesian procedure using it can be considered functionally invariant (see Section 3.1). In what 
follows we put forth some theoretical arguments and present some experimental results indicating that functional invariance may be a rather common phenomenon, even in well-known methods used for a long time by the connectionist community.

We also try to delimit the conditions that a complexity reduction method must satisfy in order to yield functional invariance. The paper focuses on the regularization methods for complexity reduction [1] and those that can be made equivalent to them. Regularization consists in adding a penalty function to the error function that regulates the complexity of the implemented network via a multiplicative factor called regularizer coefficient.

\section{The phenomenon: learned-function invariance}

We shall first define clearly the phenomenon under study, namely learned-function invariance. Let $F(X, W)$ and $G\left(X, W^{\prime}\right)$ denote the input-output functions implemented by two feedforward networks having equal number of input and output units, but different number of hidden units, and with weight vectors $W$ and $W^{\prime}$, respectively. The functional distance between $F(\bullet, W)$ and $G\left(\bullet, W^{\prime}\right)$ is defined as

$$
\operatorname{dist}\left(F(\bullet, W), G\left(\bullet, W^{\prime}\right)\right)=\frac{1}{\operatorname{Vol}(\Omega(s))} \int_{\Omega(s)}\left(F(X, W)-G\left(X, W^{\prime}\right)\right)^{2} d X
$$

when $s$ tends to infinity, $\Omega(s)$ being a cube of side $s$ in the input space.

Now, let $M(F, \lambda)$ be the optimum weight vector obtained with network $F$ by a learning method $M$ involving some complexity reduction regulated by the parameter $\lambda$. The name "method" is used here to denote an idealized algorithm, usually characterized by the minimization of an objective function, that always find global optima. If $M$ is a regularization method then

$$
M(F, \lambda)=\underset{\mathrm{W}}{\operatorname{argmin}} C(W)=\underset{\mathrm{W}}{\operatorname{argmin}} E(W)+\lambda R(W),
$$

where $E(W)$ is the standard error function and $R(W)>0$ is the regularization term.

Finally, let $\left\{F_{n}\right\}$ be a family of one hidden-layer architectures differing only in the number $n$ of hidden units. We say that the algorithm $M$ yields functional invariance for the network family $\left\{F_{n}\right\}$ if $\operatorname{dist}\left(F_{i}\left(\bullet, M\left(F_{i}, \lambda\right)\right), F_{i+1}\left(\bullet, M\left(F_{i+1}, \lambda\right)\right)\right)$ tends to zero when $i$ tends to infinity for every $\lambda>0$.

It is necessary to make some remarks about these definitions. First, we only consider global minima of $C(W)$ in the definition of $M(F, \lambda)$, and not local minima, saddle points or other points resulting from a numerical optimization of $C(W)$. Second, all the global minima of $C(W)$ must be functionally equivalent, or the distance $\operatorname{dist}\left(F_{i}\left(\bullet, M\left(F_{i}, \lambda\right)\right), F_{i+1}\left(\bullet, M\left(F_{i+1}, \lambda\right)\right)\right)$ would not be well defined. Obviously it is impossible to fulfill this condition for $\lambda=0$, but not for $\lambda>0$. This is related to the explicit exclusion of local minima from the functional invariance definition, since global and local minima, having different values of $E$, produce different outputs for the training patterns, which implies that the two minima cannot be functionally equivalent. It is possible to extend this definition using families of 
architectures that are not limited to one hidden layer of units. The only condition is that the elements of the family can be indexed in such a way that, given an arbitrary precision, for any given functional continuous mapping (from the input to the output space), there exists an index value such that architectures with higher indices can approximate the mapping with that precision. The last remark is that the definitions are independent from the training set, the only implicit requirement being that any non-empty training set would bring the functional distance limit to zero.

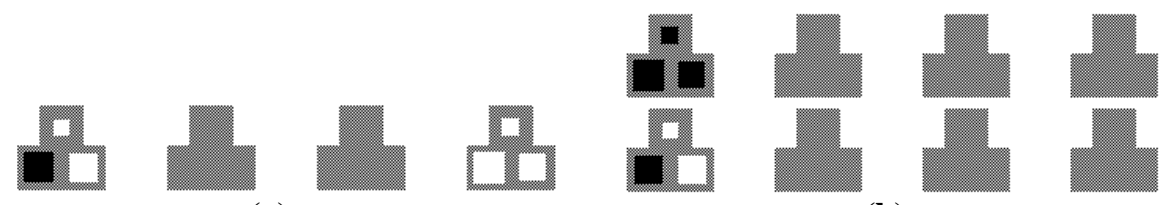

(a)

(b)

Fig. 1. The results of training a 4-HU network (a), and an 8-HU network (b) are shown. Each of the blocks in the figure contains all the weights associated to a hidden unit. Weights are represented by squares, white if their value is positive, and black if their value is negative. The size of the square is proportional to the magnitude of the weight. The top weight in the block is the weight from the hidden unit to the output unit. The weights in the bottom row correspond to the weights incoming to the hidden unit.

Figure 1 shows two networks trained with the same 20 training points, randomly drawn from the function $.3 x^{3}+.3 x^{2}+10 / 3(x+3)^{2}$ in the interval $[-1.5,1.5]$, using a deterministic algorithm to emulate learning with random weights $[5,6]$ : the mean of the weight distribution is adapted to minimize the average error over the distribution. The complexity of the function implemented by that mean is regulated via the variance of the distribution of the weights. Both the four hidden-units (HU's) network and the eight HU's network were trained using the same variance. It can be seen that the distance between the two resulting networks is null, i.e., they are functionally equivalent. Clearly, the weights of the first unit in Figure 1(a) are the same as those of the fifth unit in Figure 1(b). Moreover, the fourth unit in Figure 1(a) shows a direct correspondence with the first unit in Figure 1(b): the weights have pairwise the same magnitude, and since the signs of both the input weights and the output weights are inverted, the two units have the same functionality. It can be concluded that the two networks implement the same approximation of the desired function.

Applying the algorithm to any network with a large number of hidden units, we obtain the same units in different positions and combinations of sign inversions that produce always the same input-output function. Testing the algorithm with other variances produces other configurations that clearly converge to some function in the limit of infinite HU's. However, the closer is the variance to zero, the more difficult is the optimization, as the configurations found become more and more complex, and convergence is attained much more slowly as the number of HU's grows. This is a feature common to all the algorithms that we have explored: it is hard to check functional invariance when the complexity reduction constraints are loose. 


\section{Conditions for the appearance of invariance}

Initially, when we found functional invariance while experimenting with the random weights learning algorithm, we thought it was a rather unique phenomenon, in the sense that it was particular to the kind of weight configurations that our algorithm created, or at least, that any algorithm exhibiting functional invariance should produce weights sharing the same essential properties. However, a deeper reflection revealed that functional invariance can appear when using different algorithms, and due to very diverse reasons. Up to now, we have identified three types of algorithms corresponding to three principles in which functional invariance can be based.

\subsection{Neal's type priors}

Regularization methods can be considered under a bayesian perspective by viewing $E(W)$ and $\lambda R(W)$ as the negative log probability (disregarding some constants) of the output distribution of the function being approximated and the weight distribution, respectively. Then, $C(W)$ can be shown to be equivalent to the negative log of the posterior weight probability, and its minimization corresponds to a "maximum a posteriori" procedure.

However, the use of the same prior on two different architectures does not imply a direct relationship between the functions they implement. In fact, a prior over the weights in different architectures can induce different priors over the output functions.

Neal [4] devised a prior over the weights that, although inducing also a different prior over functions for each network, converges to a unique one as the number of hidden units tends to infinity.

Convergence of the input-output functions posterior implies convergence of its mode. Thus, a procedure optimizing this posterior may be used to obtain functional invariance. Despite this, a prior over functions in the infinite number of HU's limit is not enough to directly imply the functional invariance of the minimization of $C(W)$. In fact, this minimization finds the most probable weight vector, which does not correspond to the most probable input-output function, because there is a Jacobian determinant factor mediating the two probability densities [8], and the minimization of $C(W)$ optimizes the posterior of the weights, not that of the input-output functions.

The true bayesian procedure, however, does not consist simply in finding the mode of the weight posterior, as carried out by the usual regularization method. Instead, it takes into account the complete probability distribution to generate an answer. For example, the bayesian answer to the question "what is the best output for a given $X$ ?" under a quadratic loss function would be guessing the average of the values for that point of the posterior of the input-output functions. This involves an integration over the probability space that cancels out the Jacobian determinant, so that it is the same to integrate over the weight posterior as to integrate over the input-output function posterior. Thus, this type of answer, considered as the output of the learning algorithm, makes the complete bayesian procedure functionally invariant as we have defined it. 


\subsection{Regularizers implying a target mean function}

Input noise addition during training is equivalent to Tikhonov regularization when the number of patterns of the training set is infinite [1]. Even with finite training sets, it is equivalent to the addition of a penalization term $[5,6]$, although this is not a classical regularizer because it involves the output training patterns and depends on $E(W)$. However, the function invariance property of noise addition is better understood by taking a wider perspective. The minimization of a quadratic $E(W)$ can be viewed as an attempt to estimate with the network the mean values taken by the output patterns for a given input. Usually, there are none or very few desired values for each point in the input space. However, with input noise addition, potentially infinite patterns are available, and the expected output pattern for a given input point is [3]:

$$
m(X)=\sum_{s=1}^{n p} Y_{s} p\left(X-X_{s}\right) / \sum_{k=1}^{n p} p\left(X-X_{k}\right)
$$

where $\left\{X_{i}, Y_{i}\right\}_{i=1 . . n p}$ are the original (not noisy) training patterns and $p$ is the probability density function of the noise. We require $p(X)$ to be non zero for every $X$ in the input space, so that the randomly generated patterns cover it completely. This assures that $m(X)$ is always well-defined, since otherwise the denominator could be zero somewhere. We need $m(X)$ to be well-defined over the entire input space, because the zones of the input space that do not have desired values leave the network free to interpolate arbitrarily. Instead, if a target function is defined over all the input space, any family of networks with enough approximation power has to approximate the same function without degrees of freedom left. Thus, the fundamental condition for noise addition to be a function invariant method is the use of infinite domain probability density functions.

An important remark is that the existence and uniqueness of $m(X)$ independently of the architecture used, makes input noise addition functionally invariant in the more general sense given in Section 2, not limited to one-hidden layer architectures.

\subsection{Decomposable regularizers}

Let us now talk about regularizers for one-hidden layer networks that are additively decomposable, $R(W)=\sum_{u} r\left(\mathbf{w}_{u}\right)$, where $\mathbf{w}_{u}$ is the vector of all incoming and outcoming weights of hidden unit $u$. We require also that $r\left(\mathbf{w}_{u}\right)$ has a minimum value of zero attained when $\mathbf{w}_{u}=\mathbf{0}^{1}$. These regularizers exhibit functional invariance if there exists a threshold for $r\left(\mathbf{w}_{u}\right)$, such that when the number of units tends to infinite, all the units under this threshold tend to the minimum of $r\left(\mathbf{w}_{u}\right)$, i.e., their associated weights tend to zero.

This can be argued as follows: suppose we have a network with $n$ hidden units that has been brought to a global minimum of $C(W)$. In the limit of $n=\infty$, if we order the values of $r\left(\mathbf{w}_{u}\right)$, this sequence must tend to zero or, otherwise, $R(W)=\infty$ in the

\footnotetext{
${ }^{1}$ As a matter of fact, it would suffice that the weights from the input layer (excluding the bias unit) to $u$ were zero in the minimum of $r\left(\mathbf{w}_{u}\right)$.
} 
minimum. Thus, there exists a finite number $N$ of units whose $r\left(\mathbf{w}_{u}\right)$ is above the threshold and, therefore, are significantly non linear. The remaining $n-N$ units contribute globally with a purely linear mapping $A$ to the input of the output layer.

For a network with $n-1$ hidden units, a configuration with the same $N$ nonlinear units and all but one of the same $n-N$ linear units, reproduces the same function implemented by the $n$ HU's with a difference that tends to zero as $n$ grows. With an appropriate scaling of the linear units, the cost $C(W)$ would be the same because, on the one hand, since the $n-N-1$ units are linear, the mapping $A$ can be recovered perfectly (therefore keeping $E(W)$ ) and, on the other hand, since they are on the minimum of $r\left(\mathbf{w}_{u}\right)$, the infinitesimal scaling does not affect the regularization cost, because $\partial r / \partial \mathbf{w}_{u}=0$.

This is a global minimum of the $n-1$ HU's network. If there was a lower minimum with different nonlinear units or a different linear mapping, it would be easy to build a weight configuration in the $n$ HU's having the same $E(W)$ and the same or lower $R(W)$, which would contradict the hypothesis that the original minimum of $C(W)$ for the $n$ HU's network was global. Thus, functional invariance is guaranteed.


(a)

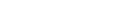
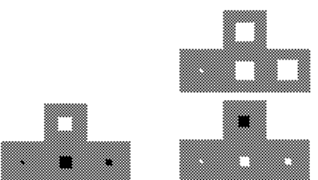

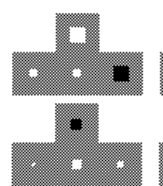

(b)

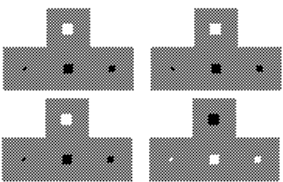

Fig. 2. Results of training a 4-HU network and a 8-HU network with 25 samples of the function $\sin \left(x_{1}+x_{2}\right)$, using a weight-decay coefficient value of .4. The resulting configurations contain replicated units that fill all the spare units available.

The deterministic algorithm to emulate learning with random weights, which triggered this work, belongs to this category. It relies on the equivalence of weight noise addition with the addition of a decomposable regularizer, which for networks with linear function activations at the linear units takes the form [6,7]:

$$
r\left(\mathbf{w}_{u}\right)=a y_{u}^{2}+b \sum_{m} w_{m u}^{2} y_{u}^{\prime 2}
$$

where $a$ and $b$ are constants, $w_{m u}$ is the weight from the hidden unit $u$ to the output unit $m$, and $y_{u}$ and $y_{u}^{\prime}$ are the activation function of $u$ and its derivative, respectively. It can be observed that this regularizer satisfies the condition of having minimum at zero only when symmetric activation functions are used.

But the most surprising example of this type of regularizer is the old good weightdecay. Careful experiments indicate that it satisfies the non-obvious condition mentioned in the first paragraph of this section. The theoretical demonstration is work still in progress. As an example, see Figure 2, where the weights resulting from training a 4-HU network and a 8-HU network with 25 samples of the function $\sin \left(x_{1}+x_{2}\right)$ are shown. Linear activation functions were used at the output units. One can see that in Figure 2(a) the first and the fourth units are replicated. In Figure 2(b), 
the same non-replicated units as in (a) appear, and the two units that were replicated in (a) appear six times in (b) but with smaller weight magnitudes. This is the scaling we were talking about before. When the number of hidden units is very large, the weight magnitudes of the replicated units become practically null, but they keep globally forming the same linear mapping.

We approximate experimentally the functional distance as the mean square distance between the outputs of two networks in a grid of 10,000 points regularly distributed in the input domain. Figure 3 shows the functional distances between architectures with different number of hidden units, minimized for several weightdecay coefficient values $\alpha$. Since for some $\alpha$ 's, some of the networks fell in local minima, in these cases we used the best minimum selected from three different random trials. It is evident that as $\alpha$ grows, all the architectures tend to produce the same results. But the most interesting observation from this graph is that, for any $\alpha>$ 0 , the distances between architectures decrease very quickly as the number of hidden units grows, and are indistinguishable from zero above 50 units. Notice that the comparisons involve networks that differ the more in the number of hidden units, the larger are the architectures. The above observation agrees with the expectation of a tendency to closer similarity for larger nets. Of course all the architectures exhibit almost the same generalization error for any positive $\alpha$, especially those above 50 HU's.

\section{Discussion}

In this paper we have put forth a definition of functional invariance, which basically states that a learning method is functionally invariant if, when applied to increasingly large networks, the output for every possible input tends to a limit, and have examined what kinds of methods possess this property. We have identified three mechanisms that can originate functional invariance:

- bayesian treatment of neural networks with weight priors that converge to a prior over functions,

- implicit definition of a mean target for the complete input space, and

- additively decomposable regularizers that produce minima with a finite number of nonlinear units in the limit of infinite units.

Examples of the first two types of mechanisms are bayesian learning with Neal's type weight priors, and input noise addition using probability density functions taking non-zero values in all their domain, respectively. Examples of the third type are the regularizer that emulates learning with random weights and classical weight-decay. There are relations between these mechanisms, but it is difficult to see a unifying principle. For example, the second type can be viewed as defining a prior over inputoutput functions, as the first type does, namely one that always concentrates the probability on a single function. However, this prior is the same for all networks using the second type of mechanism, while in the first mechanism the prior over functions is approximated only for large networks. In addition, since the target function is completely defined in the second type of mechanism, the distance to that function is 
directly minimized, whereas in the first type, averaging over the probability distributions is required to guarantee functional invariance.

There are also differences in the type of units that these mechanisms generate. The third type produces only a finite number of nonlinear units in the infinite limit, while the second gives rise to an infinite number of them. Take into account that the implicit target function can be anyone and, therefore, an infinite number of nonlinear units is required to approximate it [2].

It could seem strange that no one (up to our knowledge) had observed the functional invariance of, for example, weight-decay. However, careful optimizations are required to observe regular patterns in the weight configurations and thus see this property with sharpness. This does not mean that these results are not of practical relevance; as two different but large networks are brought moderately close to a global minimum, the functional distance between them becomes very low. The problem of falling into local minima can be significant, but their frequency using weight-decay is apparently not high. For example, in the experiment of Figure 3 comparing the functional distances of several nets with different weight-decay coefficients, among the numerous optimizations required, only three times we got a local minimum in a single trial.

Fig. 3. Evaluation of the similarity between the functions implemented by architectures with different numbers of hidden units. Values spanning a wide range of the weight-decay coefficient $\alpha$ are tested.

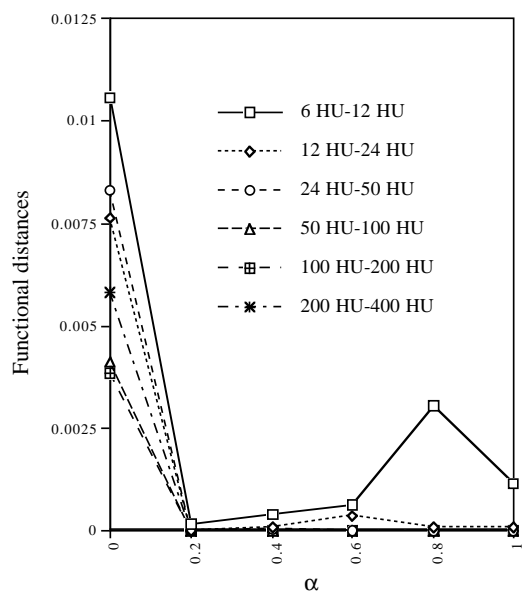

\section{References}

1. Bishop, C.M.: "Neural networks for pattern recognition". Oxford University Press, 1995.

2. Hornik, K.: "Approximation capabilities of multilayer feedforward networks". Neural Networks, Vol 4 (2), pp. 251-257, 1991.

3. Koistinen, P. and Holmstrom, L.: "Kernel regression and backpropagation training with noise", Advances in Neural Information Processing Systems 4, Morgan-Kauffman, 1992.

4. Neal, R.M.: "Bayesian learning for neural networks". Springer-Verlag, New York, 1996.

5. Ruiz de Angulo, V. and Torras, C.: "Random weights and regularization", ICANN'94, Sorrento, pp. 1456-1459, 1994.

6. Ruiz de Angulo, V. and Torras, C.: "A deterministic algorithm that emulates learning with random weights", Neurocomputing (to appear).

7. Ruiz de Angulo, V. and Torras, C.: "Architecture-independent approximation of functions", Neural Computation, Vol. 13, No. 5, pp. 1119-1135, May 2001.

8. Wolpert, D.H. (1994): "Bayesian backpropagation over I-O function rather than weights", Advances in Neural Information Processing Systems 6, Morgan Kauffman, 1999. 